\title{
WILL CIGARETTE PLAIN PACK WORK? AN EXPLORATORY STUDY IN THE UK
}

\author{
Dr. Lukman Aroean, Bournemouth University Business School, UK \\ laroean@bournemouth.ac.uk \\ Insan Syafaat MSc, Jones Lang LaSalle, Jakarta, Indonesia \\ Insan.Syafaat@ap.j1l.com
}

\section{INTRODUCTION}

Smoking has been a major public issue across the globe and considered as one of leading causes of death that kills approximately six million people a year (WHO 2012). Governments and health organizations have been constantly trying to reduce number of smokers through controlling the promotion and advertising of tobacco product (WHO 2008). In the UK, the control has begun since 1996 with the prohibition on television and radio, followed by the ban on direct marketing and sponsorship in 2005, the prohibition of the sale of tobacco product on vending machine in 2011, and the ban on the point of sale display of tobacco in large stores in 2012, which ban will take effect for small stores from 2015 (ASH 2012). As most forms of promotion and advertising are prohibited, cigarette packaging has become the remaining important promotion tool in reaching potential and current smokers (Gallopel-Morgan et al. 2012; Freeman et al. 2011). On 1 December 2012 Australia has become the first country in the world in implementing cigarette plain packaging (BBC 2012). Obviously opposing criticisms have emerged arguing that plain packaging might generate risk, such as business difficulty for retailers (Alliance of Australian Retailers 2011) and uncertainty that plain packaging would meet its aims (British American Tobacco 2012).

Standing Committee on Health (1994) defined plain packaging as a packaging without any distinctive feature or use of trade mark, is identical in appearance and colour, should be dull with un-alluring colour (with white is unacceptable because it suggests purity and cleanliness), with standardized package sizes, materials, opening methods, colours, text placement, font and size of printing. Hammond (2010) proposed that plain packaging offers three primary benefits in discouraging the use of tobacco products: increasing the effectiveness of health warnings, reducing false health beliefs about cigarettes, and reducing brand appeal especially among consumers. Would smokers reduce or stop smoking because of this? Also, although there is an increasing attention to study consumers' perception towards the product appeal of plain package, little is given to evaluate consumers' feeling about smoking from a plain pack. To address the above issues, the paper explores consumers' attitude and perception of plain cigarette packaging with three main objectives: to investigate smokers' attitude towards the appeal of plain packaging (e.g. colours, product descriptors, health warnings, and pictorial graphic design), to explore their feeling about smoking from plain pack, and to explore the impact of plain packaging towards the smokers' future smoking behaviour.

\section{DOES CIGARETTE PACK OPERATE AS PERSONALITY MARKETING TOOL?}

Brown and Williamson (1985) asserted that "a cigarette pack is the only thing you take out of your pocket 20 times a day and lay out for everyone to see. That's a lot different than buying your soap powder in generic packaging". More recently cigarette packaging mainly serves as a promotional tool that differentiates brands in the homogenous consumer product of cigarettes (Underwood, 2003). Through brand logos, colours, fonts, package materials, 
product descriptors and shapes, cigarette packaging might still be able to communicate brand image and personality (Underwood, 2003), so that smokers can associate cigarette packaging fit to their daily smoking routines (Wakefield et al 2002). While to some extent cigarette packaging might represent consumers' personal statement (Wakefield et al 2002), what are the things that enable this?

Building on the study by Philip Morris (1990), Wakefield et al (2002) found that colours on cigarette packs have an important role on generating the perceptions of consumers towards its brand appeal. Pollay and Dewhirst (2003) and Hammond and Parkinson (2009) further identified that standardized colours on cigarette packs have widely contributed to consumers' misperceptions as well as to perceptions of brand risk. Using Marlboro pack, compared to a red logo, a gold logo pack was viewed as having lower health risk and having easier-to-quit perception for adult smokers in the UK (Hammond and Parkinson, 2009). Further, smokers of certain colours on different brands were more likely to believe that their own brands might be safer, compared to smokers of uniformed colours on different brands (Mutti et al 2011). The above studies suggest that removing colours from packs (plain packaging) might significantly reduce the false beliefs.

With regard to product descriptors, removing brand elements from cigarette packs, such as brand names and logos, might result in consumers viewing cigarette packs as less appealing, having more negative expectations of cigarette taste, and rating attributes of a typical smoker of the pack less positively (Germain et al 2009). Further, Doxey and Hammond (2009) indicate that young women perceived the plain pack condition with no descriptors, for example "slim", as less likely to believe that smoking helps people stay slim. Plain packaging with the removal of product descriptors might reduce smoking appeal exposure to smokers, particularly young women (Hammond et al 2011).

Beede and Lawson (1992) suggested that plain packaging would increase consumers' awareness towards health warnings. Placing health warnings and pictorial graphic design on cigarette pack has helped students to have an improved ability to recall the health warnings and pictorial graphic designs (Goldberg et al 1999), suggesting that plain packaging might reduce smoking intension (Rootman and Flay 1995). Hammond et al (2007) identified that smokers in Canada who were exposed to large pictorial graphic designs were significantly more likely to think about the health risks of smoking. Wakefield et al (2008) found that in addition that plain cigarette packs were perceived as less attractive than the normal cigarette packs, the larger and more noticeable a health warning on a particular pack, the more likely it was to be recalled.

Interestingly disparity exists on the effect of plain pack to the feeling smokers have from plain pack smoking and to the future of their smoking intention. Moodie and Ford (2011) found that while male young smokers tend to have the same feeling when smoking from a plain pack compared to smoking from a branded pack, female smokers tend to feel worse when smoking from a plain pack. Another study involving 140 young adult smokers discovered that when smoking from a plain cigarette pack, female smokers were more likely to keep the pack out of sight, smoke less around others, eventually, think about quitting (Moodie et al., 2011).

\section{RESEARCH METHOD}


A semi structured interview is used to explore smokers' attitude and perceptions on plain pack. A purposive sampling strategy, selecting participants who will best to answer research questions and meet its objectives, is used (Saunders et al., 2009). The sample consists of 40 smokers, 13 heavy smokers (20+ cigarettes/day, WHO 2012) and 27 non-heavy smokers (<20/day), 19 of them are male and 21 females, their occupation includes students, youth worker, unemployed and manager, and their age spans from 18 to 45 years old. The semistructured interviews consisted of four parts. In the first part, we explained the participants briefly about plain cigarette packaging in the United Kingdom, the regulation, and shown a 2D photograph of plain cigarette packaging to help respondent focus on the research topic. In part two, we interviewed the participants about their perception towards product appeal of plain packaging particularly with the colour, descriptors, health warnings, and overall appeal of the pack. Next we interviewed the participants about their thoughts, feelings and argumentations about smoking from a plain pack. Finally we interviewed participants about their future smoking behavior, i.e. whether plain packaging would alter their future smoking behaviour: smoke less, or more, or consider quitting. The data were analyzed employing a grounded theory approach, which includes coding the data into concepts, identifying similarities and relationships among concepts, and extracting the main themes of the data set. In explaining the findings, the word most, majority and quite a few refer to at least more than half of the participants. When analyzing extreme statement, wherever possible we also looked into connecting them with gender and smoking intensity level.

\section{FINDINGS AND DISCUSSION}

\section{Poor appeal, but to some still likable}

Most of the respondents view, compared to normal, branded pack, the colours and the product descriptors of plain pack as giving a strong negative impression, for example "boring" (male, 24, student, heavy smokers), "emotionless" (f, 23, student, non-heavy), and "not appealing" (f, 21, waiter, non-heavy). Overall plain packaging gives poor appeal to the respondent smokers. Interestingly, while majority express negative comments with no difference found between genders, four heavy smokers gave their comments with extreme expression.

"The colour is disgusting" ( $m, 24$, heavy)

"Reminder of the death" $(f, 25$, heavy).

"It is too simple, they have just killed people's creativity in design area" ( $m, 48$, heavy);

"It does look weird, ugly, and losing its characteristic, not exciting" (m, 29, heavy).

At the opposite, four non-heavy smokers admitted that the colour of plain pack is still ok, informative, and, even, likable.

"I think it is as informative as the ones we have now" (m, 23, non-heavy)

"... made me aware of the amount of cigarettes that they would have to smoke" $(f, 28$, nonheavy).

"It looks quite alright" ( $m, 23$, non-heavy).

"Pleasing"( $f, 28$, non-heavy)

The above finding gives a hint that smoking intensity might influence perception and attitude to the plain pack.

\section{Increase health awareness, but ignorable}

Quite a few respondents acknowledged that the health warnings and pictorial graphic design on plain packaging are effective to increase their awareness of health damage. There is no indication for gender or smoking intensity to influence the views regarding health awareness.

"It pinpoints the effect of smoking tobacco" ( $f, 27$, non-heavy) 
"It is more visible since there is no other attributes" ( $m, 27$, heavy)

"The plain pack surely emphasizes both health warnings and pictorial graphic design"

(f, 27, non-heavy).

Although the respondents admit that they become aware of health warnings, they tend to think that these are ignorable, and even think the warnings are exaggerated.

"They have always been there, but has no effect on me" ( $m, 29$, heavy)

"At first it is very annoying, but later I don't care" (m, 38, non-heavy)

"People realize the effect, those warnings don't have to be exaggerated, it will not work" (f, age 31, heavy).

In sum, pack might be the closest media to smokers, but not the most powerful

" ... even though I think cigarette packaging is not the most powerful tool to educate

people about the harmful effects of smoking, it is the closest media to reach cigarette users and to which they are exposed as often as they buy a new package" (f, 25, nonheavy)

\section{Persistence: It's not the pack, but the smoking itself}

Despite the poor appeal of and negative impression caused by plain pack, most of the respondents believe smoking is still enjoyable because it's about smoking the cigarette, not the pack. Further, as most respondents persist to believe that smoking from a plain cigarette pack is as enjoyable as the current packs, they don't believe that plain packaging would alter their smoking behavior, such as smoking less or consider quitting.

"Pack is all about the place to put your cigarettes, I don't mind smoking from a bag full of lucky strike" (m, 36, non-heavy)

"In the end, it's the taste that I'm looking for, not the pack" (m, 22, heavy)

"Smoking involves enjoying the sensation of inhaling burned tobacco instead of examining what's in there on the packaging. ... because I'm addicted already? Haha and this package is just a pack. I would not change my habit ... I don't think I would quit. That's for sure. Less or more... Well it depends on the mood. Hmm ... This pack would not motivate me I guess." (f, 25, non-heavy)

Nevertheless, five non-heavy smokers, four of them female, indicated that plain pack might discourage their future smoking intention, as some of their comments below

"Definitely less, as it is making me bored, I will probably quit smoking" ( $f, 23$, nonheavy)

"Since we will look like an idiot if we smoke from a plain package, I will perhaps smoke less" (f, 28, non-heavy)

This gave a hint that plain pack might work better for non-heavy smokers than for heavy smokers.

\section{IMPLICATIONS AND CONCLUSION}

The present study extends our knowledge on whether plain pack might reduce future smoking behaviour. At the bottom line, plain pack tends to damage cigarette brands' appeal. However, although plain pack tends to increase awareness of smoking's health risk, these warnings seem to be ignorable because the on-going smokers, heavy and non-heavy, persist that enjoying smoking is not centered on the pack, but the cigarette and smoking itself. Also, although cigarette pack is closest to the smokers, its effect might not be the most powerful. There is a hint that smoking intensity (non-heavy v. heavy smokers) might moderate how smokers think and feel about plain pack. There is also a hint that smoking intensity might moderate how plain pack discourages smokers from smoking in the future. Compared to previous studies (Moodie and Ford 2011; Moodie et al 2011), the study finds no indications 
for particular gender plays a role for plain packaging influence smokers' current smoking behaviour and attitudes. Research in the future may study the impact of plain pack to potential smokers. Also, future research may study how smoking intensity plays a role in the relationship between plain pack and smokers' perception and attitude to future smoking behaviour.

There is clear indication that plain packaging might not be able to alter on-going smoker's smoking intensity up to the expected degree and the preventive effectiveness of smoking by introducing plain pack seem to be rather limited. In the future, a shift might occur from cigarette pack-associative perception and belief towards embracing natural, core characteristics of tobacco or cigarette product. More specifically, the eradication of tobacco product brands may drive smokers 'naturally' return to the original smoking state, something like when human first invented tobacco to be enjoyable to consume: a brand-less, packagingless, natural tobacco consumption culture. In other words, a smoking culture might be "back to nature".

Future research may explore the effect of plain pack in related categories such as alcohol or non-healthy food. Finally, for the purpose of reducing smoking in the future, other relevant measures might be considered in order to complement the plain packaging strategy.

\section{References}

Available upon request 